\title{
Spin-up of Be stars in the pre-main sequence phase
}

\author{
K. Stȩpień ${ }^{1,2, \star}$ \\ 1 University of Western Ontario, Department of Physics \& Astronomy, London, Ontario, Canada N6A 3K7 \\ 2 Warsaw University Observatory, Al. Ujazdowskie 4, 00-478 Warszawa, Poland
}

Received 26 September 2001 / Accepted 26 November 2001

\begin{abstract}
In an attempt to explain faster than average rotation of Be stars, a model for rotational evolution of a pre-main sequence (PMS) star with a weak primordial magnetic field was applied to stars with masses between 3 and 7 solar mass. The model takes into account the accretion of matter along the magnetic field lines, the stellar field-disk interaction and a magnetized wind. Evolutionary changes of the stellar moment of inertia are also included. The stellar mass and magnetic flux were assumed constant during the PMS evolution. The results indicate that magnetic accretion spins up a star early in its PMS life and if the star has a short PMS life time (i.e. high enough mass), it may keep faster rotation until the zero age main sequence (ZAMS). Detailed calculations show that typically a factor of two faster rotation is achieved compared to the conserved angular momentum case. This requires an intense accretion going on for a substantial fraction of the PMS phase in the presence of a surface magnetic field not exceeding $400 \mathrm{G}$. Stronger fields slow down the stellar rotation very efficiently by the magnetic field-disk locking mechanism. Low mass stars with PMS life time significantly longer than the time scale of spin down by the magnetic wind may be efficiently braked by the wind operating after the disappearance of the disk. They can retain faster than average rotation only under very special conditions. It is postulated that ZAMS progenitors of Be stars possess fossil magnetic fields with surface intensity between 40 and $400 \mathrm{G}$. The fields result in rotation rates about two times higher than those of normal stars in full agreement with the observations. If the observed Be stars have already evolved from ZAMS, their present magnetic fields should be correspondingly weaker due to the evolutionary increase of the stellar radius. ZAMS stars with magnetic fields weaker than about $40 \mathrm{G}$ should have normal rotation and those with fields significantly stronger than $400 \mathrm{G}$ should become slowly rotating Ap-Bp magnetic stars. The case of $\beta$ Cephei, with its present surface magnetic field close to $400 \mathrm{G}$, is a special case of an intermediate field, strong enough to slow down the star's rotation in the PMS phase but apparently not strong enough to develop Bp star characteristics.
\end{abstract}

Key words. stars: emission-line, Be - stars: magnetic fields - stars: pre-main sequence - stars: rotation

\section{Introduction}

Classical Be stars are distinguished from normal B dwarfs and giants by the presence of Balmer line emission. About $20 \%$ of all B stars show Be characteristics (Underhill \& Doazan 1982) with a maximum incidence around B2B3 type (Slettebak 1982). Struve (1931) suggested that the emission line spectrum is formed in a circumstellar envelope. Later observations confirmed this explanation by showing the presence of an infrared continuum excess and short wavelength-shifted UV emission lines. Measurements of the rotation velocity clearly indicate that Be stars rotate on average substantially faster than normal B stars. After analyzing the data of Slettebak (1982), Porter (1996) came to the conclusion that the equatorial velocity of the Be stars is equal to $70 \%$ of the critical velocity for which centrifugal acceleration on the equator balances gravity. A recent investigation by

\footnotetext{
* e-mail: kst@astrouw.edu.pl
}

Clark \& Steele (2000) showed that $<v \sin i>$ of the investigated Be stars is equal to $0.4 v_{\text {crit }}$ whereas it is only $0.2 v_{\text {crit }}$ for normal B stars. Here $v$ is the stellar equatorial velocity, $i$ is the inclination of the rotation axis to the line of sight and $<>$ denotes the average value. The authors adopted $v_{\text {crit }}=(2 G M / 3 R)^{1 / 2}$ after Porter (1996), with $G$ the gravity constant and $M$ and $R$ stellar mass and radius. It is not clear whether the rotation velocities of Be stars belong to a high velocity tail of a single distribution describing rotation of all B type stars or whether they form a separate velocity distribution, shifted toward faster rotation relative to normal stars. In the first case one would expect a constant ratio of the number of Be stars to the number of normal stars within the each spectral subtype. This seems not to be the case: normal B stars do not show any rapid increase in population around B2-B3 spectral type, contrary to Be stars. If the latter possibility is correct an additional mechanism spinning up some of the B stars, 
relative to others, is necessary. The present paper discusses such a mechanism.

The suggested mechanism is based on the interaction of a (weak) stellar magnetic field with the circumstellar matter in the pre-main sequence (PMS) phase of evolution. It will be shown that early type stars can be spunup by magnetic accretion from the disk surrounding the PMS star. If the magnetic field is, however, too strong, a very efficient field-disk interaction will prevent the star from fast rotation and, in fact, the star will decrease its rotation rate, compared to the conserved angular momentum (AM) case. The proposed mechanism requires that all Be stars possess fossil magnetic fields. Early observations of the Zeeman effect in these stars brought null results (Barker et al. 1981; Landstreet 1982). There were only indirect indications for the presence of a magnetic field (Fox 1993; Smith et al. 1998). Nevertheless, many models of the circumstellar disk involved weak or moderate magnetic fields (Chen \& Marlborough 1992; Porter 1997; Balona \& Kaye 1999; Smith \& Robinson 1999). Recently the first detection of a moderate magnetic field on the surface of a non-magnetic B star has been announced. Donati et al. (2001) detected a field with the surface intensity of $360 \mathrm{G}$ on $\beta$ Cephei. Although the star is not considered to be a member of Be type objects, it shows intermittent Be characteristics. This detection strengthens substantially the assumption that weak or moderate magnetic fields may also be present on other stars not possessing characteristics of the classical magnetic stars.

In the next section a model for the PMS evolution of the stellar angular velocity is described. In Sect. 3 a discussion of the input parameters is presented, the results of the calculations are given in Sect. 4 and Sect. 5 contains main conclusions.

\section{Model for AM evolution of a PMS stars with a fossil magnetic field}

The model considered here is essentially the same as used by Stępień (2000, henceforth KS) for a discussion of the evolution of AM of Ap magnetic stars in the PMS phase, except that two modifications better describing the present situation are introduced.

The angular velocity of a star possessing a magnetic field is changing during the PMS evolution because of the accretion of matter along the magnetic field lines, the magnetized stellar wind, the interaction of the stellar field with the disk and also because of the change of the stellar moment of inertia. The first three interactions can be described as torques exerted on the star. A more detailed discussion of the torques is given in KS so here only a brief resume will be given with a detailed description of the modifications introduced.

The expression for the disk torque was given by Armitage \& Clarke (1996). We assume, similarly as in $\mathrm{KS}$, that the radius of the magnetosphere (= inner radius of the disk) is equal to the corotation radius at which the Keplerian angular velocity is equal to the stellar angular velocity. A maximum efficiency of spin down occurs in such a case. The resulting relation for the torque simplifies substantially in this case and it becomes

$T_{\text {disk }}=-\frac{\mu^{2} \omega^{2}}{3 G M}$

where $\mu=B_{\text {surf }} R^{3}$ is the magnetic moment of the stellar magnetic field (here $B_{\text {surf }}$ is the surface intensity of the field), and $\omega$ is the stellar angular velocity. Deviations from this assumption will be discussed in Sect. 5. KS assumed that the fossil magnetic field of an Ap star results in $\mu=$ const. While this may be acceptable in the case of low mass stars which go through the full convection phase during which the magnetic field may be concentrated by convection in the central parts of a star, this assumption is not applicable for more massive stars which never pass a full convection phase and their evolutionary time scale is much shorter than the (non-turbulent) diffusion time scale for the magnetic field. In this case a better assumption is that of constancy of the total stellar magnetic flux, i.e. $\phi=B_{\text {surf }} R^{2}=$ const during the PMS evolutionary changes of the stellar radius (note that, keeping analogy with the definition of $\mu$, a $\pi$ factor is omitted here). We replace $\mu$ with $\phi R$ in the above equation. The disk torque does not depend on the mass of the disk as long as the mass exceeds a certain limiting value (Armitage \& Clarke 1996; Cameron et al. 1995). It is assumed here that the disk mass fulfills this condition.

The equation for the torque due to accretion was derived by KS assuming that all the AM carried with matter moving from the magnetospheric radius to the stellar surface adds to the stellar AM. In fact this is an overestimate of the AM gain because only the difference between the AM of the accreted matter and the stellar surface matter is added to the stellar AM. For slowly rotating stars with relatively strong magnetic fields, considered by KS, the radius of the magnetosphere is much larger than the stellar radius and the former approximation is justified. In the present case we have weak magnetic fields and rapid rotation. As a result the magnetosphere is not very far from the stellar surface and the specific AM of the stellar matter cannot be neglected. The resulting equation for the torque is

$T_{\mathrm{acc}}=\frac{(G M)^{2 / 3} \dot{M}_{\mathrm{acc}}}{\omega^{1 / 3}}-\omega \dot{M}_{\mathrm{acc}} R^{2}$

where $\dot{M}_{\text {acc }}$ denotes the accretion rate. The second term should be multiplied by the sine of the polar angle at which the accreted matter is deposited. We assume that the matter is deposited not far from the equator and we drop that factor for simplicity.

AM loss of a star caused by a magnetized wind is equivalent to AM carried away by the wind matter strictly corotating up to the Alfvén surface defined as a surface at which the outflow velocity equals the Alfvén velocity. The outflow velocity is usually assumed to be equal to sound velocity (e.g. Mestel 1984) or escape velocity (Kawaler 1988). The first assumption applies best to thermal winds 
Table 1. Stellar input parameters.

\begin{tabular}{ccccccc}
\hline $\begin{array}{c}M \\
\left(M_{\odot}\right)\end{array}$ & $\begin{array}{c}t_{0} \\
\text { (years })\end{array}$ & $\begin{array}{c}t_{\text {ZAMS }} \\
(\text { years })\end{array}$ & $\begin{array}{c}R_{0} \\
\left(R_{\odot}\right)\end{array}$ & $\begin{array}{c}R_{\text {ZAMS }} \\
\left(R_{\odot}\right)\end{array}$ & $\begin{array}{c}I_{0} \\
\left(M_{\odot} R_{\odot}^{2}\right)\end{array}$ & $\begin{array}{c}I_{\text {ZAMS }} \\
\left(M_{\odot} R_{\odot}^{2}\right)\end{array}$ \\
\hline 3.0 & $2.04 \times 10^{6}$ & $8.7 \times 10^{6}$ & 5.54 & 2.00 & 3.95 & 0.59 \\
4.0 & $1.01 \times 10^{6}$ & $4.3 \times 10^{6}$ & 7.34 & 2.35 & 9.40 & 1.19 \\
5.0 & $5.70 \times 10^{5}$ & $2.5 \times 10^{6}$ & 8.68 & 2.68 & 16.6 & 2.05 \\
6.0 & $3.4 \times 10^{5}$ & $1.6 \times 10^{6}$ & 10.1 & 2.96 & 27.4 & 3.18 \\
7.0 & $2.2 \times 10^{5}$ & $1.1 \times 10^{6}$ & 11.8 & 3.23 & 44.1 & 4.61 \\
\hline
\end{tabular}

of the solar type stars with hot coronae whereas the latter one seems to better describe winds in the stars considered here. Assuming that the wind velocity is equal to escape velocity and adopting a dipolar geometry for the magnetic field one obtains from the formula (9) in Stẹpień (1995)

$T_{\text {wind }}=-\frac{\omega R^{7 / 5} \phi^{4 / 5} \dot{M}_{\text {wind }}^{3 / 5}}{3(2 G M)^{1 / 5}}$,

where $\dot{M}_{\text {wind }}$ is the mass loss rate via the magnetized wind.

The final equation for the evolution of the stellar angular velocity is

$$
\begin{aligned}
\frac{\mathrm{d} \omega}{\mathrm{d} t}= & \frac{(G M)^{2 / 3} \dot{M}_{\mathrm{acc}}}{I \omega^{1 / 3}}-\frac{\phi^{2} R^{2} \omega^{2}}{3 I G M} \\
& -\frac{\omega R^{7 / 5} \phi^{4 / 5} \dot{M}_{\mathrm{wind}}^{3 / 5}}{3 I(2 G M)^{1 / 5}}-\frac{\omega \dot{M}_{\mathrm{acc}} R^{2}}{I}-\frac{\omega \mathrm{d} I}{I \mathrm{~d} t},
\end{aligned}
$$

where $I=k^{2} M R^{2}$ is the stellar moment of inertia with $k^{2}$ a radius of gyration.

In the next section the input parameters appropriate for Be stars will be discussed.

\section{Input parameters}

It is generally accepted that Herbig AeBe stars are the progenitors of the intermediate mass stars. They are surrounded by disks, show the presence of intense accretion and stellar outflows. Accretion rates, determined for Herbig AeBe stars for the masses considered here are within the interval $10^{-8}-10^{-6} M_{\odot} /$ year (Hillenbrand et al. 1992) but there exist several apparently disk-less stars, which indicates that disks may be present only throughout a part of the PMS life (de Winter et al. 1997). The estimate of mass loss of Herbig AeBe stars due to a stellar wind is of the order of $10^{-8} M_{\odot} /$ year (Catala 1989; Palla 1991). Accretion rates and mass loss rates due to a stellar wind will be varied around the observed ranges, with the notion that the particular choice of a specific value cannot violate the assumption of the constant stellar mass, i.e. the net mass loss/gain must be small compared to the stellar mass.

The third parameter needed to solve the problem is the strength of the stellar surface magnetic field. Similarly as in $\mathrm{KS}$ a unit of the magnetic field is taken as equivalent to the $1 \mathrm{kG}$ field on the surface of a $2 R_{\odot}$ star. The magnetic flux of such a field is equal to $1.9 \times 10^{25}$ in cgs units. Note, however, that, contrary to the assumption made by $\mathrm{KS}$ that the magnetic field varies as $R^{-3}$, the assumption of the constant magnetic flux requires that the field varies as $R^{-2}$.

Detailed models of PMS stars of intermediate mass were computed by Iben (1965), by Palla \& Stahler (1993) and very recently by Siess et al. (2000). The latest models show that the PMS phase of these stars lasts somewhat longer that in models obtained by Palla \& Stahler (1993) but not as long as in computations by Iben (1965). Siess et al. (2000) made available on Internet very detailed tables of several stellar parameters as functions of time, including the stellar radius and moment of inertia. These data are used throughout the paper.

The computations of PMS evolution obtained by Siess et al. (2000) start very early, when the star is still within a protostellar cloud. The initial time for the present calculations was adopted as the moment when the star crosses the stellar birth line (Palla \& Stahler 1993). Stars with masses 3 and $4 M_{\odot}$ cross the line twice, so the second time was taken as the initial time. In case of stars with 6 and 7 solar masses, the birth line approaches very closely the ZAMS, leaving little room for a PMS phase. The initial time for these stars was taken, rather arbitrarily, somewhat beyond the birth line where stellar moment of inertia was still about ten times larger than on ZAMS i.e. similarly as in the case of less massive stars. It will be shown later that the particular choice of this instant does not influence the main results. Detailed stellar input parameters were taken from Siess et al. (2000) and are given in Table 1.

To allow for changes of the relative importance of the three processes changing the stellar AM and described by the first three terms on the right hand side of Eq. (4), efficiency factors were introduced, defined in the same way as in $\mathrm{KS}$

$$
\begin{aligned}
& \dot{M}_{\text {acc }}=k_{\text {acc }} \times 10^{-8} M_{\odot} / \text { year } \\
& \phi=k_{\text {mag }} \times 1.9 \times 10^{25} \\
& \dot{M}_{\text {wind }}=k_{\text {wind }} \times 10^{-8} M_{\odot} / \text { year } .
\end{aligned}
$$

They can be regarded as direct measures of the respective parameters entering Eq. (4), i.e. $k_{\text {acc }}=10$ corresponds to the accretion rate $\dot{M}_{\text {acc }}=10^{-7}$, or $k_{\text {mag }}=0.1$ to the surface magnetic field of $100 \mathrm{G}$ on the surface of a $2 R_{\odot}$ star. The surface intensity of the stellar magnetic field on a star with radius $R$ can be calculated from the following formula

$B_{\text {surf }}=\frac{4000 k_{\mathrm{mag}}}{\left(R / R_{\odot}\right)^{2}} \quad \mathrm{G}$. 
The three coefficients will be used in the form of a "vector" [ $k_{\text {acc }}, k_{\text {mag }}$ and $\left.k_{\text {wind }}\right]$ when discussing different solutions.

Equation (4) was solved using the 4th order RungeKutta method with the interpolated values of the stellar radius, moment of inertia and its derivative calculated from the tables provided by Siess et al. (2000) using the cubic spline (Press et al. 1986). Rigid rotation was adopted following the assumption that the primordial magnetic field is able to enforce it.

The results of computations will be compared with periods resulting from conservation of $\mathrm{AM}$, henceforth called "normal". These periods do not correspond exactly to periods of non-magnetic stars undergoing the same PMS evolution because disk accretion also spins up a non-magnetic star. The spin up via a boundary layer is, however, usually considerably less efficient than in case of magnetospheric accretion and is not described correctly by the accretion term in Eq. (4). The comparison of the observed $v \sin i$ of Herbig AeBe stars with their young counterparts on the MS showed that, depending on the degree of AM mixing inside the stars, both compared groups have identical or very similar AM (Böhm \& Catala 1995). Errors connected with neglecting any AM change of non-magnetic stars should therefore be much less than the difference between the ZAMS rotation period of a normal and a Be star. Variations of the thus defined normal period result solely from the changes of the stellar moment of inertia during its PMS evolution.

\section{Results}

\subsection{Stars with masses of $7 M_{\odot}$}

We start with the most massive star for which the necessary data are available (Siess et al. 2000). The PMS life time of the star is less than one million years (Table 1 ). For a weak or moderate magnetic field with $k_{\text {mag }} \leq 1$, and $k_{\text {wind }} \leq 10$, the time scale for spin down via a magnetized wind is of the order of $10^{7}$ years i.e. much longer than the PMS life time of the $7 M_{\odot}$ star. The influence of such a wind on the stellar AM can safely be neglected in this case. We will therefore not discuss the wind for the present case but, instead, we will concentrate on the discussion of the other two mechanisms influencing the stellar AM.

As an illustration of a typical evolution of the rotation period under the influence of all the considered mechanisms, Fig. 1 shows two families of curves (dashed and dotted lines). They both start with the same initial rotation periods of 3,5 and 7 days but they describe different evolutionary tracks of the stellar AM: the dotted lines show spin up of the star whereas the dashed lines show spin down relative to the star with conserved AM. A solid line shows the evolution of the rotation period when $\mathrm{AM}$ is conserved (with only one initial value of 5 days, to avoid crowding) which can be considered as a reference line reflecting the variation of the stellar moment of inertia. It was assumed that the disk exists during the whole PMS phase and the values of the efficiency factors were

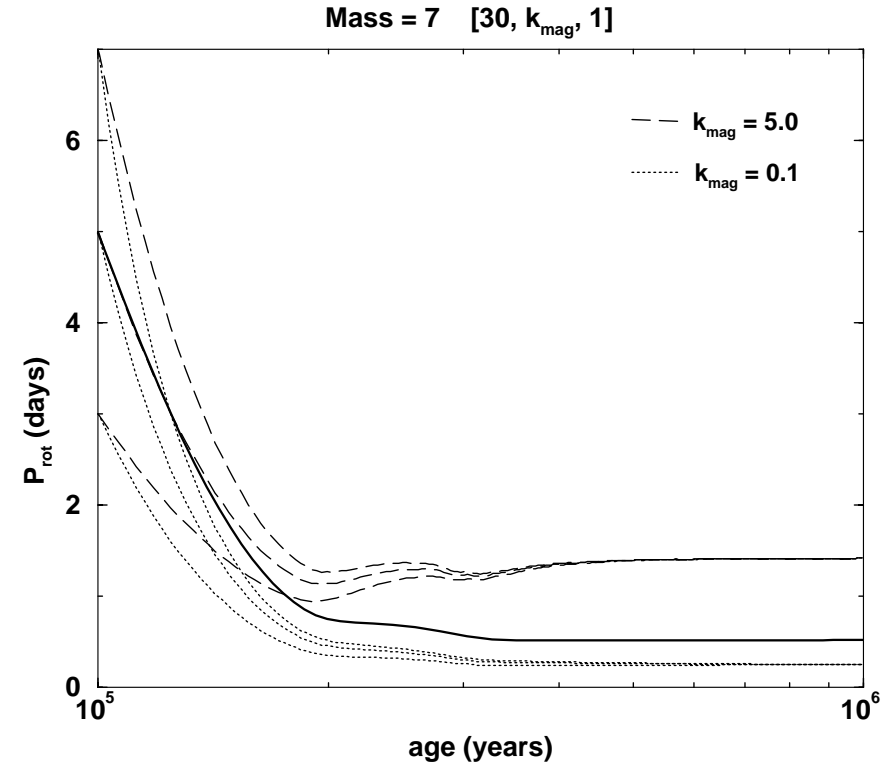

Fig. 1. Evolution of a rotation period of a star with $7 M_{\odot}$ during the PMS phase. Normal, non-magnetic case (i.e. with the conserved AM) is shown as a solid line with the initial rotation period of 5 days. The evolutionary tracks of the rotation period of a star with a strong magnetic field, when intense spin down relative to the normal star occurs, are shown as dashed lines and those of a star with a weak magnetic field, when the star is spun up, as dotted lines. Note a fast merging of curves corresponding to different initial periods to a single rotation period depending only on the interaction of the stellar field with circumstellar matter. The vector gives the adopted values of the efficiency coefficients $k$, see definition (5).

adjusted to enhance spin up and spin down. The two families of curves were obtained with the same values of $k_{\text {acc }}$ and $k_{\text {wind }}$ but with different values of $k_{\text {mag }}$, as shown in the figure. The ZAMS values for the dotted curves are close to 0.25 of a day and for the dashed curves 1.42 day. They should be compared with the value of 0.52 of a day given by the solid line. The zero point of this figure and all others was shifted arbitrarily to $10^{5}$ years.

All the mechanisms influencing the stellar AM which we have considered tend to decrease differences among initial values of the rotation period (compare Eq. (4)). As a result a memory of the initial period is quickly wiped out by the interaction of the stellar magnetic field with the circumstellar matter and the ZAMS rotation period depends solely on this interaction, as it is seen in Fig. 1 (see also KS). A precise value of the initial rotation period of a star is therefore unimportant. In the following, only one initial value, equal to 5 days, will be used.

Figure 2 demonstrates the influence of a change in the stellar magnetic field on the evolution of the rotation period. Dashed lines give the evolution of the stellar rotation period for five different values of $k_{\text {mag }}$ between 5 and 0.1 (from the top to bottom). The two bottom curves, corresponding to $k_{\mathrm{mag}}=0.3$ and 0.1 , are practically identical. A solid line describes again a normal case. For the $7 M_{\odot}$ ZAMS star, $k_{\text {mag }}=1$ corresponds to a surface magnetic 


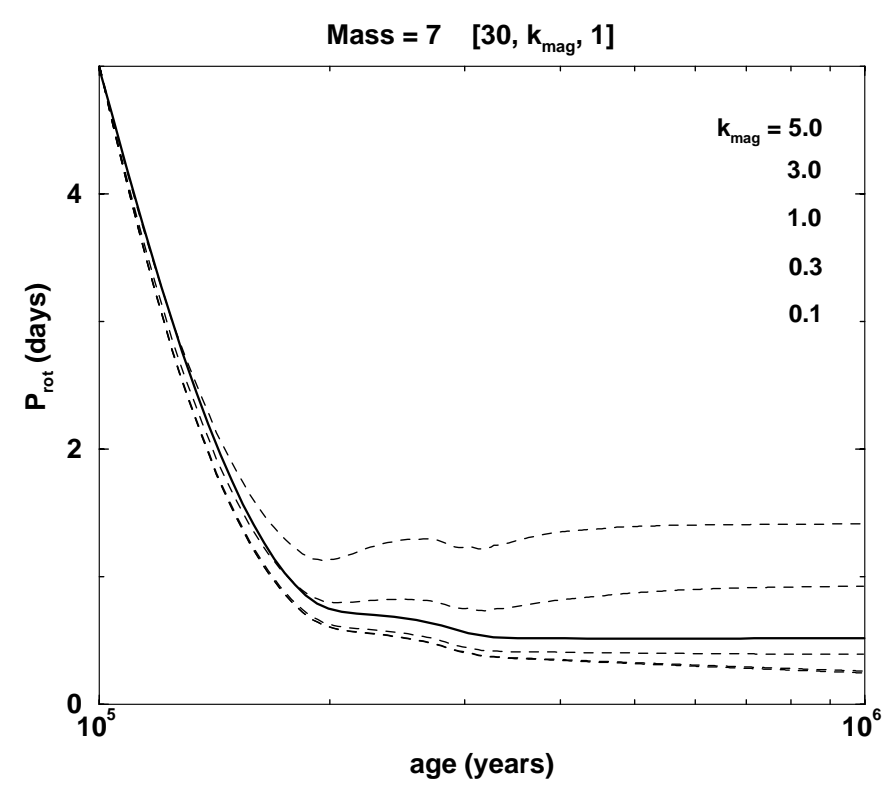

Fig. 2. The influence of the strength of the stellar magnetic field on the AM evolution of a $7 M_{\odot}$ star in the PMS phase. The solid line describes a normal, non-magnetic case, when stellar AM is conserved. Dashed lines describe the evolution of the stellar rotation period for five different values of the surface magnetic field, as shown by the values of $k_{\text {mag }}$ from the top to the bottom (two lowest curves over-lie almost exactly one another). The curves illustrate the nonlinear response of the AM gain/loss mechanism to the magnetic field. For weak magnetic fields spin up occurs but for stronger fields the processes braking down rotation become more efficient and the star spins down.

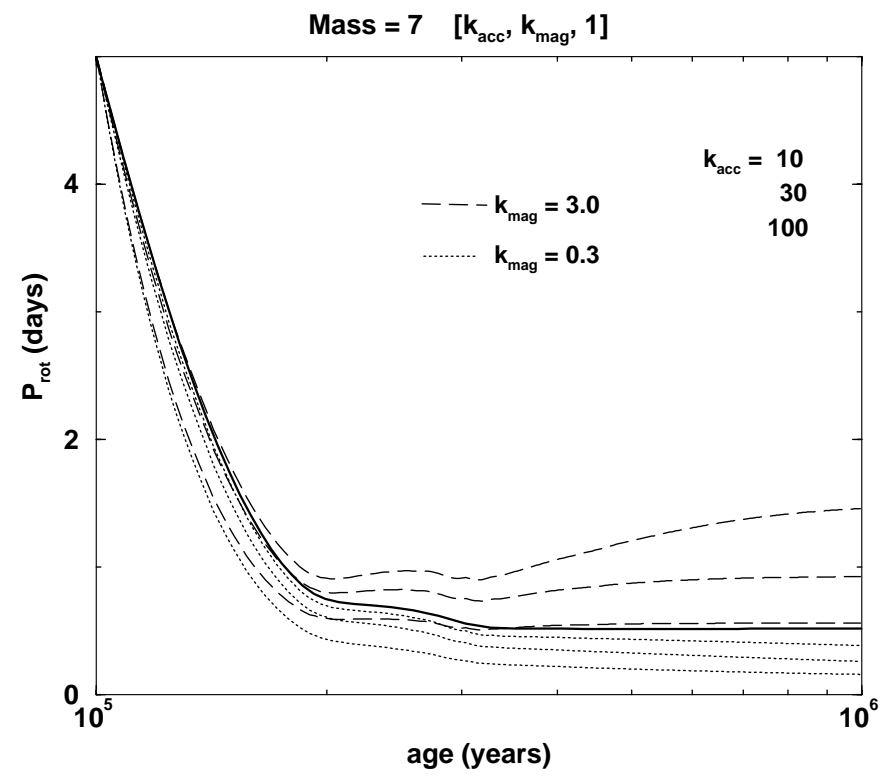

Fig. 3. The influence of the accretion rate on the AM evolution of a $7 M_{\odot}$ star in the PMS phase. The solid line shows a normal, non-magnetic case, when stellar AM is conserved. Families of the dashed and dotted lines describe the evolution of the rotation period of a star with strong and weak magnetic field, respectively (see the values of $k_{\mathrm{mag}}$ ), for three different values of the accretion rate, as given by the values of $k_{\text {acc }}$ from the top to bottom.

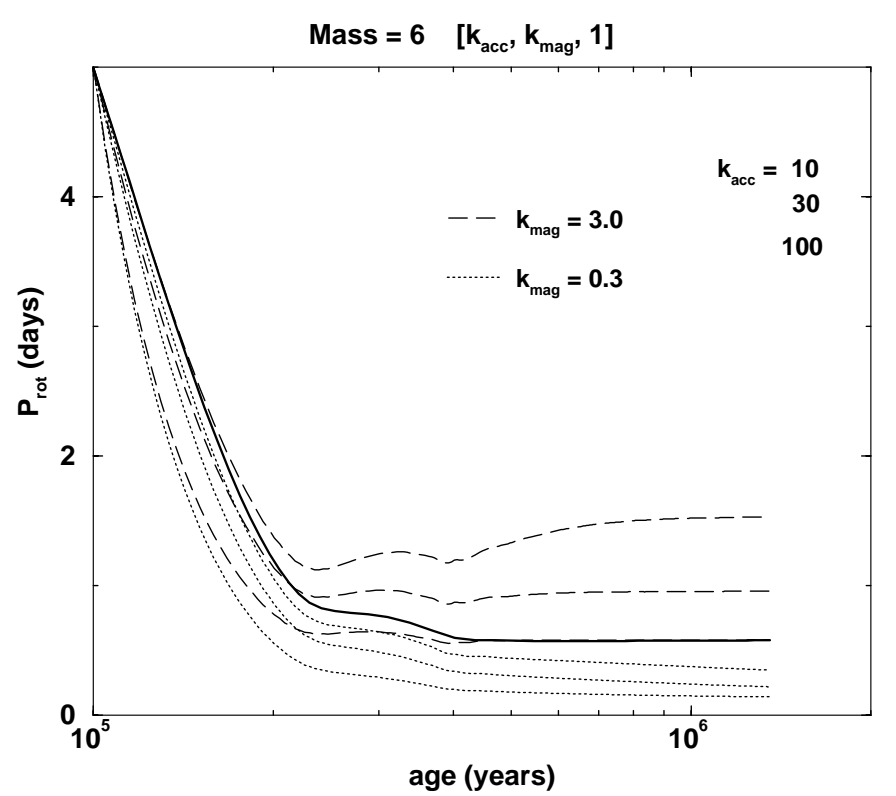

Fig. 4. The same as in Fig. 3 but for a $6 M_{\odot}$ star.

field of about $400 \mathrm{G}$ (Eq. (6)). As we see, such a field can still spin up a star to about $2 / 3$ of the normal period (see the third dashed line from the top) but still stronger magnetic fields result in a spin down of the star. Values of $k_{\text {mag }}$ between 0.1 and 0.3 (corresponding to $40-120 \mathrm{G}$ ) give the values of the ZAMS rotation periods about two times shorter than normal, as required by observations (Clark \& Steele 2000). Still lower values of $k_{\text {mag }}$ cannot be considered because Eq. (4) loses its applicability when the radius of the magnetosphere approaches the stellar radius. We can only speculate that the corresponding curves should approach the solid line from below. The accretion rate and mass loss due to the wind were not varied. We conclude from the figure that the AM change is a strongly nonlinear function of the intensity of the surface magnetic field. For weak magnetic fields the star is spun up with the maximum efficiency obtained for $k_{\mathrm{mag}} \approx 0.1-0.3$ but further increase of the field intensity leads to a less efficient spin up and finally to a slow down. This is the result of the change of the mutual importance of the terms in Eq. (4) with changing intensity of the magnetic field. Note that the first three terms in Eq. (4) depend on very different powers of the magnetic field (0,2 and 4/5). In particular, strong magnetic fields increase dramatically the role of the field-disk interaction, described by the second term. As a result, a sort of locking of the stellar rotation period to some equilibrium value occurs on a relatively short time scale (decreasing as $B^{-2}$ ) and then the period is kept essentially constant as it is seen in Figs. 1 and 2 .

The influence of varying the accretion rates is shown in Fig. 3. Here, besides a solid line describing the rotation period of a normal star, two families of curves are shown for two values of $k_{\text {mag }}$ equal to 0.3 and 3 (dotted and dashed lines, respectively). Accretion rates vary between $10^{-7}$ and $10^{-6} M_{\odot} /$ year. It is seen that stronger accretion results in shorter ZAMS periods, as intuitively 
expected. Nevertheless, the curves for different values of $k_{\text {acc }}$ lie relatively close to one another, which indicates that the influence of the variable accretion rate is generally less important than that of the varying magnetic field. Spin up can occur only for sufficiently weak magnetic fields (dotted lines) - a conclusion reached also from Fig. 2.

We conclude that the strength of the magnetic field is the primary factor determining whether a star spins up or spins down during its PMS phase of evolution. For surface magnetic fields smaller than about $150 \mathrm{G}$ (on ZAMS) almost any reasonable accretion rate results in a significant spin up. If a star has a surface field of the order of $400 \mathrm{G}$, it needs a very intense accretion, of the order of $10^{-6} M_{\odot} /$ year, for a substantial part of its PMS life, to reach a ZAMS rotation rate characteristic of a Be star. Still stronger magnetic fields always result in a spin down and a possible formation of a Bp star (see KS).

The above results were obtained assuming that a disk exists during the whole PMS phase of stellar evolution. Calculations were also carried out for a range of disk life times shorter than that but they showed that the results are essentially insensitive to the disk life time. As long as the disk life time exceeds $10 \%$ of the PMS phase the ZAMS period practically does not change. This is easily understood from Fig. 1. The spinning up of a star takes place very early - within the first few $\times 10^{5}$ years and then the rotation period follows approximately the variations of the stellar moment of inertia. The situation changes, of course, when the disk exists for a significantly shorter time than $10 \%$ of the PMS phase but we will not consider such cases.

\subsection{Stars with masses of 6 and $5 M_{\odot}$}

Stars with masses of 6 and $5 M_{\odot}$ behave very similarly to the $7 M_{\odot}$ star except that their PMS life times are correspondingly longer (see Table 1). Figure 4 shows the evolution of the rotation period for two values of $k_{\text {mag }}$ and a range of $k_{\text {acc }}$. Here, the ZAMS rotation period of a normal star (shown as a solid line) is equal to 0.58 of a day. The dotted lines give the ZAMS rotation periods for a weak field case between 0.22 and 0.35 of a day i.e. again about two times shorter than normal.

For stars of 7 and $6 M_{\odot}$ the influence of the magnetized wind is negligible (see above) but for a $5 M_{\odot}$ star the PMS life time increases to about 2 million years whereas the slow down time scale for the wind decreases significantly, mainly due to a decrease of the stellar moment of inertia (Table 1). In the weak field case the wind time scale is still longer than that but both scales can become comparable for a strong magnetic field with $k_{\mathrm{mag}}=3$, particularly if the mass loss due to the wind is large. Figure 5 demonstrates the effect of such a wind. Here, again, the dashed curves correspond to a strong field case and the dotted lines to a weak field case. Curves from the top to bottom within each family correspond to different $k_{\text {acc }}$, as indicated. Note that the maximum considered

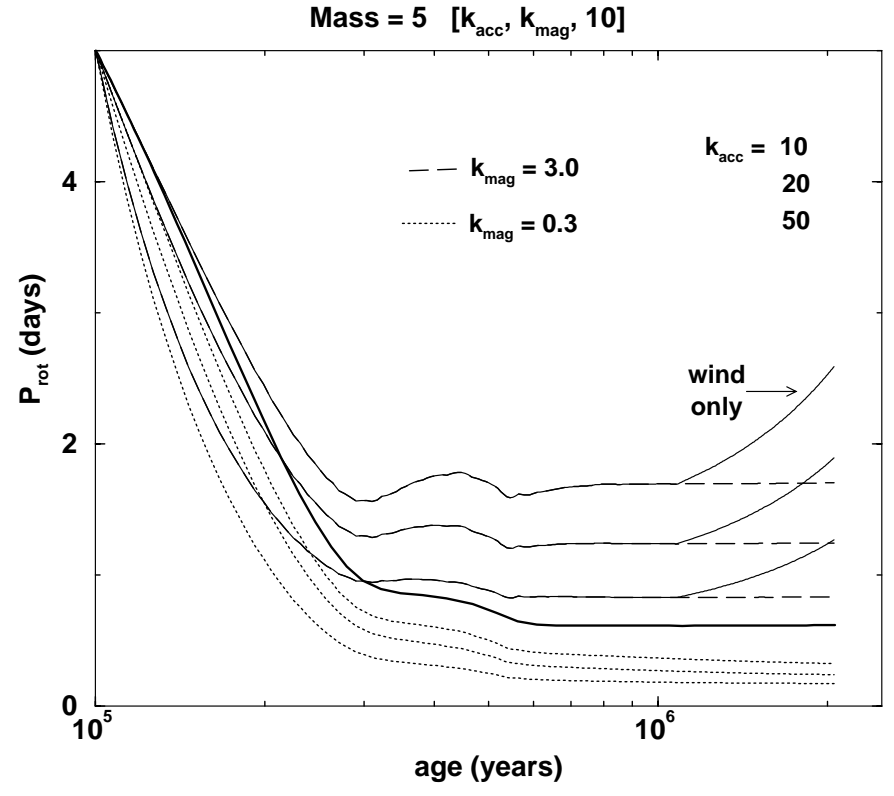

Fig. 5. Evolution of the rotation period of a $5 M_{\odot}$ star with strong and weak magnetic field (dashed and dotted curves, respectively). Curves within each family from the top to bottom correspond to different values of $k_{\text {acc }}$, as indicated. Light solid lines, partly overlying dashed cures, describe the period evolution when the disk is switched off after the first half of the stellar PMS life and only a magnetized wind operates. In the weak field case (dotted lines) the influence of the wind is negligible.

accretion rate is now lower than in the case of more massive stars to avoid a violation of the constant mass assumption. In the presence of a disk, even the relatively strong wind is unable to significantly change the rotation period. This is shown by the dashed curves which go nearly horizontally in Fig. 5. They correspond to the case when the disk exists for all the PMS phase. If, however, the disk is dispersed earlier, the situation may be different. Nothing changes, of course, when the wind disappears simultaneously with the disk but if it persists for the rest of the PMS phase, it can operate very efficiently, as is shown by light solid lines. Winds in Herbig AeBe stars are probably driven by the stars themselves and not by the disks as it is in case of $\mathrm{T}$ Tauri stars (Nisini et al. 1995) so winds can exist even when the disks are dispersed (they still exist on the main sequence). Similar calculations for $k_{\mathrm{mag}}=$ 0.3 (dotted lines) confirm that the influence of winds in a weak field case is still negligible. The resulting curves (not plotted) are indistinguishable from the dotted lines. In the intermediate case of $k_{\text {mag }}=1$ (not shown in the figure) an appreciable increase of the rotation period occurs which effectively cancels the previous spin up. In all calculations the disk was switched off after the first half of the PMS phase. We conclude that while a spin up is obtained for essentially any combination of parameters in the case of a weak field (of the order of $200 \mathrm{G}$ or less on the ZAMS), $5 M_{\odot}$ stars with intermediate fields (of the order of $500-$ $600 \mathrm{G}$ ) can be spun up only for strong accretion during 


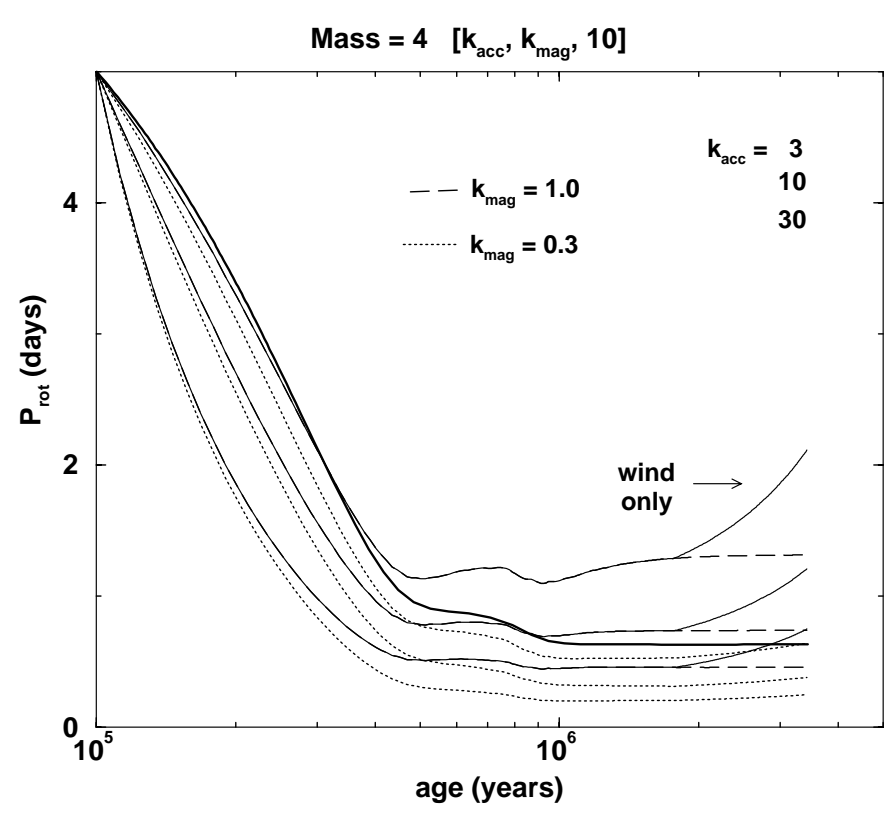

Fig. 6. The same as in Fig. 5 but for a $4 M_{\odot}$ star. The wind influences now also the weak field curves turning them slightly upwards.

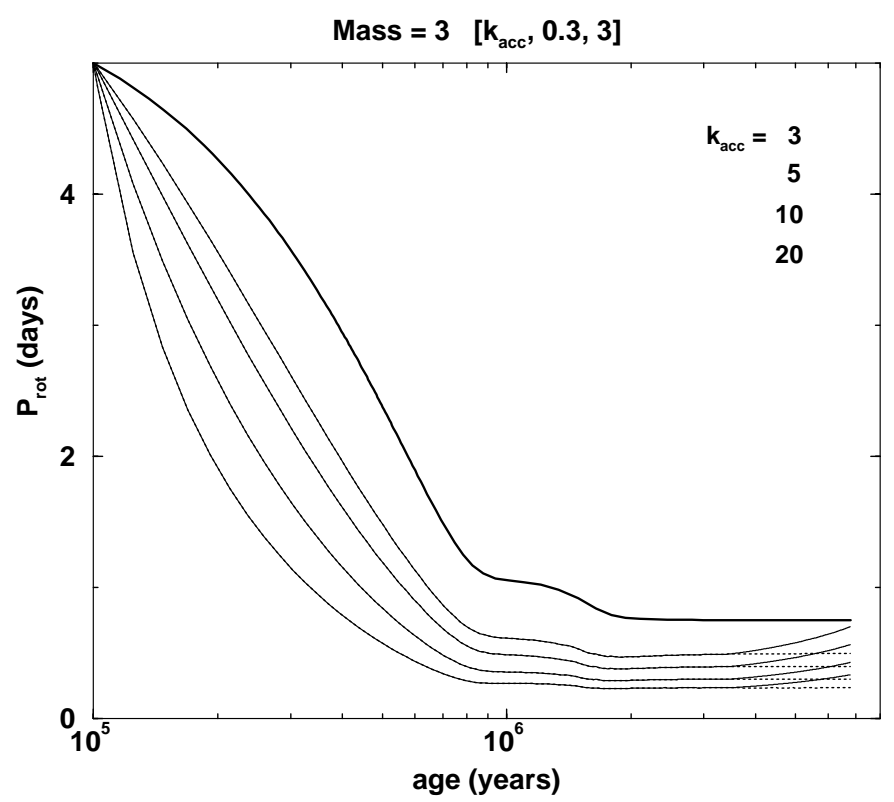

Fig. 7. Evolution of the rotation period of a $3 M_{\odot}$ star. Only the weak field case is shown with four different accretion rates. The influence of a stellar wind acting on the star during the last, disk-less half of its PMS life is shown with the light solid lines overlying partly the dotted lines. Dotted curves correspond to the case when the disk survives until ZAMS.

the whole PMS phase of evolution. If the disk disappears earlier, they will reach the ZAMS as normal or slightly spun down stars.

\subsection{Stars with masses of 4 and $3 M_{\odot}$}

Figure 6 shows that the case of a $4 M_{\odot}$ is not much different from the $5 M_{\odot}$ except that the PMS life time is still longer so the accretion rates taken for the calculations must be still lower. Knowing that the wind can efficiently spin down the star with a strong enough magnetic field only the case of a weak and an intermediate magnetic field are displayed in Fig. 6. We see that the wind operating for the last half of the stellar PMS life can spin down the star significantly even for $k_{\mathrm{mag}}=1.0$ (solid light curves). The wind has an appreciable effect even in the weak field case as is indicated by a slight turn up of the dotted lines. The highest lying dotted line shows in fact no spin up at all at the ZAMS. This means that not all stars with weak magnetic fields will be spun up. Those with a moderate, or low accretion rate and with a disk existing only over a part of the PMS phase will have normal rotation on ZAMS. Accretion rates of at least $10^{-7}$ are needed to spin up sufficiently a $4 M_{\odot}$ star with the ZAMS magnetic field of about $250 \mathrm{G}$ (which corresponds to $k_{\mathrm{mag}}=0.3$ ). Because the maximum accretion rate is limited by the constant mass assumption, this requirement narrows substantially the range of accretion rates producing fast rotating stars to $1-2 \times 10^{-7} M_{\odot} /$ year. Higher accretion rates would result in an unacceptable increase of the stellar mass but they are also not in agreement with observations which show a decrease of the accretion rate of Herbig AeBe stars with decreasing mass (Hillenbrand et al. 1992).

The PMS life time of a $3 M_{\odot}$ star is nearly 7 million years, i.e. almost an order of magnitude more than that of a $7 M_{\odot}$ star whereas the slow down time scale of the wind is about one order of magnitude shorter. This gives relatively more time for a wind to slow down the stellar rotation when the disk exists only for a part of the PMS phase. Figure 7 shows, as in the case of a $4 M_{\odot}$ star, that the accretion rate has to be rather strong, i.e. $1-2 \times 10^{-7} M_{\odot} /$ year in the presence of a magnetic field not exceeding $330 \mathrm{G}$ on the ZAMS if the star is to reach ZAMS with a rotation rate about two times faster than a normal star. For disks existing during the whole PMS phase the magnetic field can be somewhat stronger if the accretion rate is close to $2 \times 10^{-7} M_{\odot} /$ year.

\section{Discussion and conclusions}

The mechanism proposed for a spin up of intermediate mass stars in the PMS phase is based on a very robust process: when a star possessing a magnetic field accretes matter from an accretion disk, the magnetospheric accretion will efficiently spin up the star. If the interaction with the circumstellar matter can be stopped at the right moment, the star preserves its fast rotation till ZAMS. For more massive stars this happens quite naturally because of their short PMS life time. The sufficient condition for them to rotate rapidly on ZAMS is the presence of a weak, or moderate, stellar magnetic field and the existence of an accretion disk for at least $10 \%$ of the PMS phase. For less massive stars additional conditions must be fulfilled which prevent the star from dispersing its excess AM gained in the early phase of the PMS evolution; the accretion disk with a high enough accretion rate must exist for nearly 
whole PMS phase or its earlier disappearance must be accompanied by a simultaneous disappearance of the stellar wind. Too strong a magnetic field results in a spin down of a star, independent of its mass.

The model considered is based on several simplifying assumptions. First, it is assumed that the accretion and magnetic field-disk interaction is stationary (Ghosh \& Lamb 1978; Lovelace et al. 1995). However, this assumption is dependent upon the properties of the disk, particularly the diffusivity, and in some cases the interaction can take the form of a series of unsteady episodes with a time scale of months (Goodson et al. 1999; Goodson \& Winglee 1999). For the time scales considered in the present paper the details of the star-disk interaction can safely be neglected and the process can be approximated by a stationary interaction with appropriate time-averaged values for the accretion rate and disk torque. It was also assumed that the radius of the magnetosphere is always equal to the corotation radius, which produces a maximum torque exerted by the disk. Small deviations from this coincidence can be taken into account by an appropriate modification of the respective efficiency factors $k$ (see Eq. (5)). A significant deviation from this assumption can take place, e.g. for extremely weak magnetic fields, when the magnetosphere becomes strongly distorted by the massive disk due to its inner edge approach to the star closer than the corotation radius. Such a situation is not correctly described by the present model and it was excluded from the considerations. Sometimes the opposite situation can occur when the magnetosphere extends significantly further than the corotation radius. This happens when the disk mass decreases below some critical value and as a result, the stellar magnetic field quickly disperses the remainder of the disk (Armitage \& Clarke 1996; Cameron et al. 1995; Mestel 1999). As we see, both these assumptions do not significantly influence the spin up mechanism. The proposed mechanism is also insensitive to the choice of the initial moment for the stellar AM evolution. To check this, some calculations were carried out for the initial moment taken halfway between that given in Table 1 and ZAMS. Stars are at this moment already past the rapid decrease of their moment of inertia, which then remains nearly constant till the ZAMS. As long as the initial rotation period was not too close to the critical period, a rapid spin up always occurred in the first phase of AM evolution and the ZAMS period was roughly in the same proportion to the normal period as in the calculations presented here. This result agrees well with the previous calculations by KS, obtained for significantly shorter PMS life times and smaller variations of stellar moment of inertia, which showed the same effect of a rapid initial spin up of weakly magnetic stars.

The basic results of the present investigation show that the sufficient spin up, leading to a rotation rate about two times faster than in the nonmagnetic (normal) case, takes place in the first couple of $10^{5}$ years of the PMS evolution, if the star possesses a fossil magnetic field with the ZAMS surface intensity of 40-400 G. Because the PMS phase of the most massive stars considered here is of the same order, all such stars possessing the required magnetic field, should become rapid rotators with an enhanced likelihood of becoming Be stars. The fast increase of the PMS life time with decreasing mass imposes more and more stringent conditions for the AM evolution resulting in rapidly rotating ZAMS stars. This suggests that a decrease of the incidence of Be stars among stars of a given spectral type is expected for less massive stars, as observed. The mechanism is not restricted to B type stars only. As earlier calculations by KS showed, stars with masses as low as $1.5-2 M_{\odot}$ can also, under very special circumstances, reach the ZAMS with rotation substantially faster than average. Also in these cases the necessary condition for a star to become a rapid rotator is a presence of a fossil magnetic field with the ZAMS surface intensity of a few hundred Gauss. The observations show that a small fraction of $\mathrm{A}$ and $\mathrm{F}$ stars are fast rotating analogies of $\mathrm{Be}$ stars (Slettebak 1982; Abt et al. 1997). Stars more massive than $7 M_{\odot}$ mass were not considered in the present paper because of lack of the necessary input data, but one can speculate that when the PMS life time approaches $10^{5}$ years the spin up mechanism may not have enough time to operate and, again, a substantial spin up can occur only under special conditions. That should decrease the occurrence of rapidly rotating stars with increasing mass past the maximum around $7-8 M_{\odot}$, in a qualitative agreement with the observations. It is, however, not clear to what extend the disk approximation used in the present paper can be applied to thick envelopes surrounding massive stars till ZAMS.

The limits for the magnetic field of rapidly rotating stars obtained here (40-400 G) apply to ZAMS stars. The observations show that most (if not all) of the $\mathrm{Be}$ stars have already evolved away from ZAMS and several reached a giant phase. The evolutionary expansion results in a proportional decrease of the surface magnetic field. Assuming that a typical radius of a Be star is two times larger than its ZAMS radius, the expected surface field is $10-100 \mathrm{G}$ if the magnetic flux is preserved. If, instead, the magnetic dipole moment is preserved, the expected intensity will be still lower by a factor of two. Such intensity is, unfortunately, beyond the present threshold of observational detection.

A very interesting case is that of $\beta$ Cephei for which Donati et al. (2001) detected a surface magnetic field of $360 \pm 30 \mathrm{G}$. The star is a known prototype of pulsating stars and does not belong to classical Be stars although it shows episodes of Be-type activity. With $P_{\text {rot }}=12$ days the star rotates significantly slower than normal stars of a similar age. Its mass is $12 M_{\odot}$, far beyond the most massive stars considered here. We cannot therefore apply the quantitative results of the present investigation to this star. Nevertheless, we can compare it qualitatively with the $7 M_{\odot}$ mass considered here. The present radius of $\beta$ Cephei is equal to $7 R_{\odot}$ (Donati et al. 2001) hence its magnetic flux corresponds to $k_{\mathrm{mag}}=4.5$ in our units. This corresponds to a strong field case and results in a spin 
down of a star by a factor of about 3 compared to the normal star (Fig. 2). Applying this number to $\beta$ Cep and assuming a typical ZAMS rotation period of 1-1.5 day for a normal $12 M_{\odot}$ star we obtain 3-4 days for the expected ZAMS rotation period of $\beta$ Cep. This estimate agrees well with the conclusion of Donati et al. (2001) that $\beta$ Cep must have gone a significant brake down during the PMS phase of evolution. They argue that the present rotation period of the star is too long to result from the normal ZAMS rotation period even if one takes into account a possible AM loss due to the mass loss on the MS in the presence of the magnetic field. The magnetic field of $\beta$ Cep is apparently not strong enough for developing abundance anomalies typical of magnetic stars.

To summarize, the main results of the paper indicate that all intermediate mass stars with primordial magnetic fields quickly lose memory of the initial rotation period during their PMS evolution. Their ZAMS periods are the result of an interaction of the stellar magnetic field with the circumstellar environment. Accretion along the magnetic field lines from a disk spins up a star in early phases of the PMS evolution, which may result in a faster than normal rotation on ZAMS. Such stars can become Be stars. It is postulated that all Be stars possess magnetic fields of the right order. The spin up requires surface magnetic fields in the range of about $40-400 \mathrm{G}$ on ZAMS. If the Be phenomenon is developed in more advanced evolutionary phases, the present intensity of the magnetic field on the surface of Be stars should be of the order of $100 \mathrm{G}$ or less.

Acknowledgements. I thank Dr. John Landstreet for inviting me to the University of Western Ontario and for hospitality. My thanks are also due to Michael Marlborough for an enlightening discussion on Be stars. His comments and the remarks of Dr. R. D. Robinson, the referee, helped to improve the paper. This work has been supported in part by the Natural Sciences and Engineering Research Council of Canada. A partial support from the KBN grant 5P03D 00621 is also acknowledged.

\section{References}

Abt, H. A., Tan, H., \& Zhou, H. 1997, ApJ, 487, 365 Armitage, P. J., \& Clarke, C. J. 1996, MNRAS, 280, 458

Balona, L. A., \& Kaye, A. B. 1999, ApJ, 521, 407

Barker, P. K., Landstreet, J. D., Marlborough, J. M., Thompson, I., \& Maza, J. 1981, ApJ, 250, 300

Böhm, T., \& Catala, C. 1995, A\&A, 301, 155
Borra, E. F., Landstreet, J. D., \& Mestel, L. 1982, Ann. Rev. Astr. Ap., 20, 191

Borra, E. F., Beaulieu, A., Brousseau, D., \& Shelton, I. 1985, A\&A, 149, 266

Bouvier, J., Covino, E., Kovo, O., et al. 1995, A\&A, 299, 89

Cameron, A. C., \& Campbell, C. G. 1993, A\&A, 274, 309

Cameron, A. C., Campbell, C. G., \& Quaintrell, H. 1995, A\&A, 298, 133

Catala, C. 1989, in Low mass star formation and pre-MainSequence evolution, ed. Bo. Reipurth (ESO, München), 471

Catala, C., \& Kunasz, P. B. 1987, A\&A, 174, 158

Chen, H., \& Marlborough, J. M. 1992, ApJ, 397, 242

Clark, J. S., \& Steele, I. A. 2000, A\&AS, 141, 65

Donati, J.-F., Wade, G. A., Babel, J., et al. 2001, MNRAS, in press

Fox, G. K. 1993, MNRAS, 260, 525

Hillenbrand, L. A., Strom, S. E., Vrba, F. J., \& Keene, J. 1992, ApJ, 397, 613

Ghosh, P., \& Lamb, F. K. 1978, ApJ, 223, L83

Goodson, A. P., Böm, K.-H., \& Winglee, R. M. 1999, ApJ, 524, 142

Goodson, A. P., \& Winglee, R. M. 1999, ApJ, 524, 159

Iben, I. 1965, ApJ, 141, 993

Kawaler, S. D. 1988, ApJ, 333, 236

Landstreet, J. D. 1982, ApJ, 258, 639

Lovelace, R. V. E., Romanova, M. M., \& Bisnovatyi-Kogan, G. S. 1995, MNRAS, 275, 244

Mestel, L. 1984, in Cool Stars, Stellar Systems, and the Sun, ed. S. L. Baliunas, \& L. Hartmann (Springer, Berlin), 49

Mestel, L. 1999, Stellar magnetism (Oxford Univ. Press, Oxford), 383

Nisini, B., Milillo, P., Saraceno, P., \& Vitoli, F. 1995, A\&A, 302, 169

Palla, F. 1991, in Fragmentation of molecular clouds and star formation, ed. K. Falgerone et al. (Kluwer, Dordrecht), 331

Palla, F., \& Stahler, S. W. 1993, ApJ, 418, 414

Porter, J. M. 1996, MNRAS, 280, L31

Porter, J. M. 1997, A\&A, 324, 597

Press, W. H., Teukolsky, S. A., Vetterling, W. T., \& Flannery, B. P. 1986, Numerical Recipes (Cambridge University Press, Cambridge)

Smith, M. A., Robinson, R. D., \& Corbet, R. H. O. 1998, ApJ, 503,887

Smith, M. A., \& Robinson, R. D. 1998, ApJ, 517, 866

Siess, L., Dufour, E., \& Forestini, M. 2000, A\&A, 358, 593

Slettebak, A. 1982, ApJS, 50, 55

Stępień, K. 1995, MNRAS, 274, 1019

Stȩpień, K. 2000, A\&A, 353, 227 (KS)

Struve, O. 1931, ApJ, 73, 94

Underhill, A., \& Doazan, V. 1982, B Stars with and without Emission Lines, NASA SP-456

de Winter, D., Koulis, C., Thé, P. S., et al. 1997, A\&AS, 121, 223 